\title{
Chronic Exercise Training Improved Aortic Endothelial and Mitochondrial Function via an AMPK $\alpha 2$-Dependent Manner
}

OPEN ACCESS

Edited by:

Mingyi Wang,

National Institutes of Health, USA

Reviewed by:

Youyang Zhao,

University of Illinois at Chicago, USA

Maria Pikilidou,

AHEPA University Hospital, Greece

*Correspondence:

Youyi Zhang

zhangyy@bjmu.edu.cn

Pingjin Gao

gaopingjin@sibs.ac.cn

Specialty section:

This article was submitted to

Vascular Physiology,

a section of the journal

Frontiers in Physiology

Received: 11 September 2016

Accepted: 05 December 2016

Published: 21 December 2016

Citation:

Chen X, An X, Chen D, Ye M, Shen W, Han W, Zhang Y and Gao P (2016) Chronic Exercise Training Improved Aortic Endothelial and Mitochondrial Function via an AMPKa2-Dependent Manner. Front. Physiol. 7:631 doi: 10.3389/fphys.2016.00631

\author{
Xiaohui Chen ${ }^{1}$, Xiangbo $\mathrm{An}^{2}$, Dongrui Chen ${ }^{3,4}$, Maoqing $\mathrm{Ye}^{3,4}$, Weili Shen ${ }^{3,4}$, \\ Weiqing Han ${ }^{3,4}$, Youyi Zhang ${ }^{2 *}$ and Pingjin Gao ${ }^{1,3,4 *}$
}

${ }^{1}$ Laboratory of Vascular Biology and Key Laboratory of Stem Cell Biology, Institute of Health Sciences, Shanghai Institutes for Biological Sciences, Chinese Academy of Sciences, University of Chinese Academy of Sciences, Shanghai, China, ${ }^{2}$ Institute of Vascular Medicine, Peking University Third Hospital, Beijing, China, ${ }^{3}$ Shanghai Key Laboratory of Hypertension, Ruijin Hospital, Shanghai Jiao Tong University School of Medicine, Shanghai, China, ${ }^{4}$ Shanghai Institute of Hypertension, Shanghai, China

Chronic exercise training is known to protect the vasculature; however, the underlying mechanisms remain obscure. The present study hypothesized that exercise may improve aortic endothelial and mitochondrial function through an adenosine monophosphate-activated protein kinase $\alpha 2$ (AMPK $\alpha 2$ )-dependent manner. Ten-week-old AMPK $\alpha 2$ knockout (AMPK $\alpha 2^{-/-}$) mice and age-matched wild-type (WT) mice were subjected to daily treadmill running for 6 weeks, and the thoracic aorta from these mice were used for further examination. Our results showed that exercise significantly promoted vasodilatation and increased expression and phosphorylation of endothelial nitric oxide synthase (eNOS), concomitant with increased AMPK $\alpha 2$ expression in WT mice. These effects were not observed in $A M P K \alpha 2^{-/-}$mice. Furthermore, exercise training increased thoracic aortic mitochondrial content as indicated by increased Complex I and mitochondrial DNA (mtDNA) in WT mice but not in AMPK $\alpha 2^{-1-}$ mice. This may be caused by decreased mitochondrial autophagy since the expression of $\mathrm{BH} 3$ domain-containing BCL2 family members BNIP3-like (BNIP3L) and LC3B were decreased in WT mice with exercise. And these changes were absent with AMPK $\alpha 2$ deletion in mice. Importantly, exercise increased the expression of manganous superoxide dismutase (MnSOD) and catalase, suggesting that mitochondrial antioxidative capacity was increased. Notably, the improved antioxidative capacity was lost in AMPK $\alpha 2^{-/-}$mice with exercise. In conclusion, this study illustrated that AMPK $\alpha 2$ plays a critical role in exercise-related vascular protection via increasing endothelial and mitochondrial function in the artery.

Keywords: AMPK, exercise, vascular, endothelial function, mitochondrial function

\section{INTRODUCTION}

It is well documented that exercise training can effectively prevent cardiovascular risk factors such as obesity, hypertension, and diabetes in the long term (Stewart, 2002). For example, it has been shown that exercise improved vascular endothelial function in hypertensive animal models (Kumral et al., 2016) and patients with coronary artery disease (Hambrecht et al., 2000). In vessel 
samples from animals with exercise, the expression and Ser ${ }^{1177}$ phosphorylation of endothelial nitric oxide synthase (eNOS) were increased, whereas the oxidative stress was decreased. These changes lead to increased $\mathrm{NO}$ availability and improved vascular function (Kojda et al., 2001; Hambrecht et al., 2003; Adams et al., 2005). However, the mechanisms by which exercise exerts these beneficial effects on the vasculature are little known.

Adenosine monophosphate-activated protein kinase (AMPK) is a serine/threonine kinase consisting of $\alpha, \beta$, and $\gamma$ subunits. The $\beta$ and $\gamma$ regulatory subunits maintain the stability of the kinase, and the $\alpha$ subunit possesses catalytic activity (Steinberg and Kemp, 2009). In particular, two isoforms of AMPK $\alpha$, $\mathrm{AMPK} \alpha 1$, and $\mathrm{AMPK} \alpha 2$ are both expressed in endothelial cells and in smooth muscle cells (Goirand et al., 2007). Furthermore, it has been shown that AMPK $\alpha 2$ is increased during exercise in skeletal muscle cells (Magnoni et al., 2014), and the increased $A M P K \alpha 2$ can regulate gene and protein expression through direct interaction with the nucleus (McGee et al., 2003; Jørgensen et al., 2006). Recent studies suggest that AMPK has a much more important role in the vasculature as it activates and phosphorylates endothelial nitric oxide synthase (eNOS) (Morrow et al., 2003), protects endothelial cells against oxidative stress (Schulz et al., 2008) and prevents vascular smooth muscle proliferation (Nagata et al., 2004). These results may suggest a protective role of AMPK in the vascular system.

Mitochondria are mobile organelles that exist in dynamic networks. To maintain a healthy population of mitochondria, the content of mitochondria is critically regulated by biogenesis, fusion-fission, and autophagy. These regulations ultimately determine the quantity, quality, and function of mitochondria, thereby contributing to cell function (Yan et al., 2012). It has been shown that maintaining mitochondrial content and functional network is crucially important for proper function of both endothelial cells and vascular smooth muscle cells (Salabei and Hill, 2013; Szewczyk et al., 2015). As expected, the abnormal content and network regulation of mitochondria lead to various cardiovascular diseases, such as diabetic vascular dysfunction (Pangare and Makino, 2012) and hypertension (Jin et al., 2011). It has been shown that exercise can increase mitochondrial content and function in skeletal muscle (Russell et al., 2014). In addition, mitochondrial antioxidant enzymes can reduce the damaging effects of reactive oxygen species (ROS, Tang et al., 2014). For example, mitochondrial manganese superoxide dismutase (MnSOD) deficiency aggravated age-dependent vascular dysfunction and increased mitochondrial oxidative stress (Wenzel et al., 2008). Over-expressing mitochondria MnSOD in mice attenuated angiotensin II (Ang-II) induced hypertension (Dikalova et al., 2010). However, whether exercise training could improve mitochondrial function in the vasculature remains unclear. In the present study, we hypothesize that chronic exercise training may improve endothelial function and mitochondrial function in aortas and that $\mathrm{AMPK} \alpha 2$ may contribute these protective effects by mediating the expression of the corresponding proteins.

\section{MATERIALS AND METHODS}

\section{Animals and Exercise Protocol}

All animal treatment complied with the Guide for the Care and Use of Laboratory Animals published by the US National Institutes of Health (NIH Publication No. 85-23, revised 1996). All animal procedures were approved in accordance with the institutional guidelines established by the Committee of Ethics on Animal Experiments at the Chinese Academy of Sciences. Wild type (WT) mice were provided by the Institute of Laboratory Animal Science of Peking Union Medical College. AMPK $\alpha 2$ knockout (AMPK $\alpha 2^{-/-}$) mice were kindly provided by Dr. Benoit Viollet (Department of Endocrinology, Metabolism and Cancer, Institute Cochin, University Paris Descartes, Paris, France) and bred in a specific pathogen-free environment. Male $\mathrm{AMPK} \alpha 2^{-/-}$and WT mice were both with $\mathrm{C} 57 \mathrm{BL} / 6 \mathrm{~J}$ genetic background. All mice were 2 months old with a mean body weight of $18 \pm 2 \mathrm{~g}$ at the start of the experiment.

After allowing acclimatization to their housing and the treadmill, WT mice $(n=20)$, and AMPK $\alpha 2^{-/-}$mice $(n=$ 20) were randomly divided into two groups: the control group and the training group, with 10 mice in each group. Mice in the training group ran on the treadmill for $90 \mathrm{~min} /$ day at 9.0 meters/min (0\% grade), 5 days/week for 6 weeks (Fernando et al., 1993). Body weight, heart rate and systolic/diastolic blood pressure were assessed in all animals. After $12 \mathrm{~h}$ of the last training, mice were anesthetized of pentobarbital $(5 \mathrm{mg} / 100 \mathrm{~g})$ with an intraperitoneal injection and sacrificed.

\section{Western Blot Analysis}

The thoracic aortas were dissected out and immersed in liquid nitrogen immediately. Then the frozen tissues were lysed in RIPA (Radio Immunoprecipitation Assay) buffer containing $150 \mathrm{mM} \mathrm{NaCl}, 50 \mathrm{mM}$ Tris ( $\mathrm{pH} 7.4$ ), $1 \%$ sodium deoxycholate, $1 \%$ Triton $\mathrm{X}-10,0.1 \%$ SDS, protease inhibitor (sodium fluoride, sodium orthovanadate, leupeptin, EDTA) (Beyotime, Haimen, China). After sonication on ice for $30 \mathrm{~min}$ and centrifugation at $12000 \mathrm{rpm}$ for $20 \mathrm{~min}$ at $4^{\circ} \mathrm{C}$, the supernatant was collected for Western blotting as previously described ( $\mathrm{Li}$ et al., 2012). The primary antibodies were as follows: anti-MnSOD (ABclonal, MA, USA), anti-AMPK $\alpha 2$ (Abcam, Cambridge, England), anti-phospho-AMPK $\alpha 1 / \alpha 2$ (Thr ${ }^{172}$ ), anti-BNIP3L (BH3 domain-containing BCL2 family members BNIP3-like) (Bioworld, St. Louis, Park, USA), antieNOS, anti-phospho-eNOS (Ser ${ }^{1177}$ ) (BD Biosciences, NJ, UK), anti-Complex I, anti-PGC-1 $\alpha$ (peroxisome proliferator-activated receptor gamma coactivator 1 alpha), anti-Drp1(dynamin related protein 1), anti-Mfn1 (mitofusin 1), anti-LC3, anti-catalase, antiGAPDH (Santa Cruz, CA, USA), anti-mTOR (mammalian target of rapamycin), anti-phospho-mTOR ( $\operatorname{Ser}^{2448}$ ) (Sigma-Aldrich, St. Louis, MO, USA). Immunoreactive bands were highlighted by electrochemiluminescence (ECL) technology and quantified by densitometry using imaging software (Image Jversion 1.46, $\mathrm{NIH}$, Maryland, USA). The individual values were originally expressed as a percentage of a target protein and an internal protein standard (GAPDH) (target protein content/GAPDH content) and then expressed as a fold change of the normal 
WT control group (target protein content/GAPDH content) value.

\section{Immunofluorescence}

The paraffin sections were deparaffinized by dimethylbenzene and rehydrated by graded alcohol. Antigen retrieval was processed by citric acid buffer ( $\mathrm{pH} 6.0$ ) for $5 \mathrm{~min}$ at $100^{\circ} \mathrm{C}$. Then the slides were incubated in hydrogen peroxide for $10 \mathrm{~min}$ and were blocked in TBST (tris-buffered saline and tween) containing 5\% Bovine Serum Albumin at room temperature for $30 \mathrm{~min}$. Some sections were subsequently incubated with $300 \mathrm{nM}$ MitoTracker Green (Invitrogen, CA, USA) at room temperature for $30 \mathrm{~min}$. Other sections were incubated at $4^{\circ} \mathrm{C}$ overnight with antibodies against AMPK $\alpha 2$ (1:100, Abcam, Cambridge, England), fluorescent anti-rabbit secondary antibody at a 1:400 dilution for $30 \mathrm{~min}$, and then nucleus dye 4,6-diamidino-2phenylindole (DAPI) for $3 \mathrm{~min}$. All images were taken by using a Zeiss Pascal LSM 710 confocal microscope (Germany). Fluorescence intensity was analyzed with Image Pro Plus in three independent samples.

\section{Mitochondrial DNA Copy Number}

Genomic DNA of the thoracic aorta tissue was extracted by using UniversalGen DNA Kit(Cwbiotech, Beijing, China). The mitochondrial $(\mathrm{mt})$ copy number was analyzed by realtime PCR (ABI 7900 Real Time PCR System; Foster City, CA) as previously described (Ray Hamidie et al., 2015), through the relative value of mitochondrial and nuclear DNA (mt:nuclear DNA) which reflects the amounts of mitochondria per cell. The mitochondrial DNA (mtDNA) forward primer was CCTAGGGATAACAGCGCAAT $\left(5^{\prime}-3^{\prime}\right)$ and the reverse primer was ATCGTTGAACAAACGAACCA. The nuclear DNA (nDNA) forward primer was AGAGCTCTGCGGGTACATCT and the reverse primer was CATCAGTGACGGTGCCTTAC. QPCR were performed in a real time PCR system: the PCR began with $95^{\circ} \mathrm{C}$ denaturation for $30 \mathrm{~s}$ followed by 40 cycles of $95^{\circ} \mathrm{C}$ denaturation for $5 \mathrm{~s}$, and annealing and elongation for $34 \mathrm{~s}$ at $60^{\circ} \mathrm{C}$. Samples were assayed in triplicate. Cycle threshold (CT) was used for data analysis, and CT (nDNA)-CT (mtDNA) or $\Delta \mathrm{CT}$ was used to reflect the difference in CT values. Results were expressed as the copy number of mtDNA per cell, $2 \times 2^{-\Delta C T}$.

\section{Thoracic Aorta Ring Assay}

Mice thoracic aortas were separated, cleared of fat and connective tissues, cut into $2-3 \mathrm{~mm}$ rings, and fixed on isometric force transducers (Danish Myo Technology Model 610 M, Denmark) in a $5 \mathrm{ml}$ organ bath, and aerated with $95 \% \mathrm{O}_{2}$ and $5 \%$ $\mathrm{CO}_{2}$ under an initial resting tension of $2.5 \mathrm{mN}$ (Zhou et al., 2014). Force was recorded in a PowerLab/8sp data acquisition system (A.D. Instruments, Castle Hill, Australia). After $1 \mathrm{~h}$ of incubation in oxygenated Krebs' medium (containing: $\mathrm{KCl}$ $4.7 \mathrm{mmol} / \mathrm{L}, \mathrm{NaCl} 118 \mathrm{mmol} / \mathrm{L}, \mathrm{CaCl}_{2} 2.5 \mathrm{mmol} / \mathrm{L}, \mathrm{KH}_{2} \mathrm{PO}_{4} 1.2$ $\mathrm{mmol} / \mathrm{L}, \mathrm{MgSO}_{4} 1.2 \mathrm{mmol} / \mathrm{L}$, glucose $11 \mathrm{mmol} / \mathrm{L}$ and $\mathrm{NaHCO}_{3}$ $25 \mathrm{mmol} / \mathrm{L}$ ) at $\mathrm{pH} 7.4$ and $37^{\circ} \mathrm{C}$, rings contractility was tested 3 times by high $\mathrm{K}^{+}$mediums $(60 \mathrm{mM} \mathrm{KCI})$ to stabilize the contraction. Cumulative response curve of phenylephrine $\left(10^{-8}\right.$ to $10^{-4} \mathrm{~mol} / \mathrm{L}$ ) was performed to assess the vasoconstriction response and cumulative concentration-response curves of acetylcholine $\left(10^{-8}\right.$ to $\left.10^{-4} \mathrm{~mol} / \mathrm{L}\right)$ were constructed with a phenylephrine pre-contraction $(3 \mu \mathrm{mol} / \mathrm{L})$.

\section{Statistical Analysis}

All values are reported as means $\pm \mathrm{SD}$. Comparison of groups involved Student's unpaired two tailed $t$-test or two-way ANOVA with the Bonferroni test for post-hoc analysis (SigmaPlot Software, San Jose, CA, USA). $P<0.05$ was considered statistically significant.

\section{RESULTS}

\section{The Protein Expression and Phosphorylation of AMPK $\alpha 2$ were Increased in Mice Aorta with Chronic Exercise}

Firstly, there was no significant difference of body weight and systolic blood pressure in WT and AMPK $\alpha 2^{-/-}$mice pre and post exercise as shown in Table S1. Table S1 also showed that heart rate was decreased in the exercise group by $17.7 \%$, which was comparable in $\mathrm{WT}$ and $\mathrm{AMPK} \alpha 2^{-/-}$mice.

Next, we evaluated whether chronic exercise would have any effect on AMPK $\alpha 2$ expression. As shown in Figure 1A, AMPK $\alpha 2$ expression was dramatically increased in the aorta after chronic exercise training in immunofluorescence staining, although the overall vascular architecture had no significant difference in the four groups of mice in H\&E staining (Figure S1). This was consistent with western blot results showing that exercise training induced a significant increase in aortic AMPK $\alpha 2$ protein expression by $31 \%$ in WT mice (Figure 1B). Furthermore, it was found that phosphor-AMPK $\alpha$ (p-AMPK $\alpha$ ) (T172) was also significantly increased in the aorta of exercised mice by using phosphor-specific antibody against both $\alpha 1$ and $\alpha 2$ isoforms of AMPK. In AMPK $\alpha 2^{-/-}$mice, the protein of AMPK $\alpha 2$ was not detectable due to gene knockout, and p-AMPK $\alpha$ (T172) had similar basal levels to WT mice but showed no increase in response to exercise. These results indicated that exercise induced a significant increase in the expression and phosphorylaton of AMPK $\alpha 2$ in the aorta.

\section{AMPK $\alpha 2$ Deficiency Decreased Vasodilation and eNOS of Aorta in Exercise} We then analyzed whether exercise would have any beneficial effect on vasodilation. As shown in Figure 2A, the vascular relaxation to acetylcholine was decreased in aorta rings from AMPK $\alpha 2^{-/-}$mice compared with age-matched wild type mice, indicating that AMPK $\alpha 2$ was involved in NO-dependent vasodilation. Importantly, the improvement of vasodilation was significantly lower in AMPK $\alpha 2^{-/-}$mice compared with WT mice, although exercise increased the vasorelaxation ability of the aorta in both WT and AMPKa $2^{-/-}$mice (Figure 2A). These results indicated that AMPK $\alpha 2$ played an important role in exercise-related vasorelaxation. In contrast, the vasoconstriction of aortas responding to phenylephrine was similar among the 
A
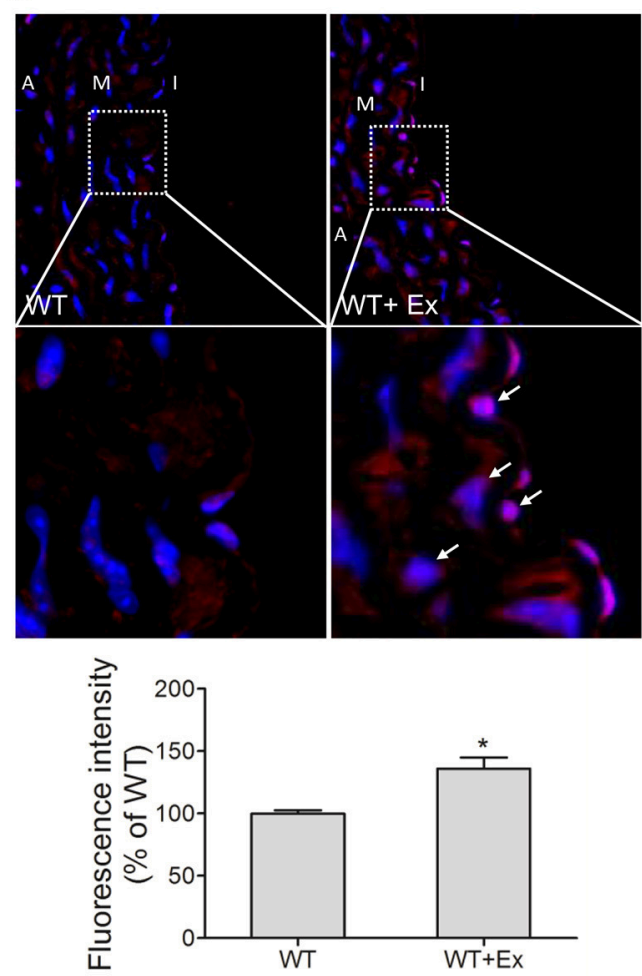

B
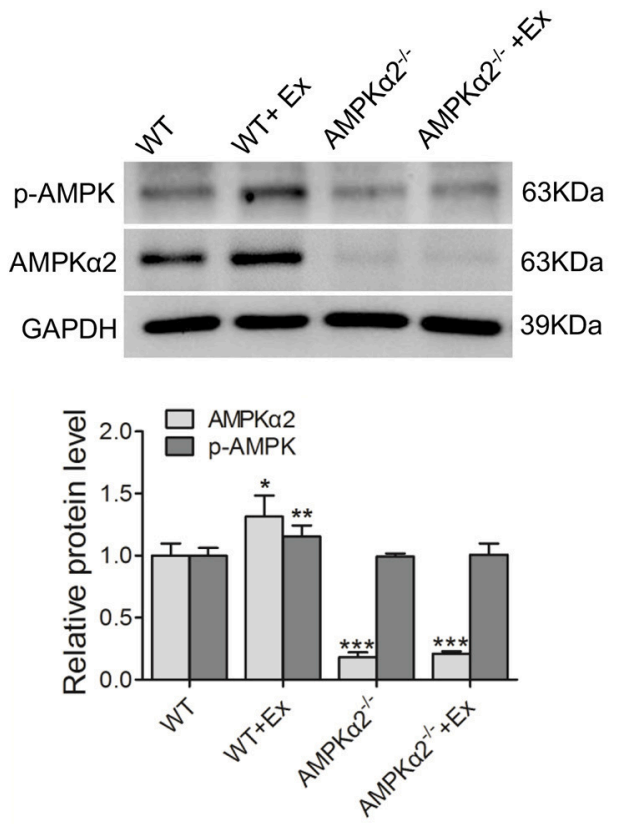

FIGURE 1 | Exercise training increased thoracic aortic AMPK $\alpha 2$ activity. (A) Representative immunofluorescence images and fluorescence intensity analysis showing the expression of AMPK $\alpha 2$ in aorta, in which fluorescence-positive cells were stained in red (arrow) and nuclei were counterstained with DAPI (blue). (B) Representative western blot images and summarized data showing the expression of p-AMPK $\alpha 2$ and AMPK $\alpha 2$ of aorta from WT and AMPK $\alpha 2$ knockout mice with or without exercise. The protein levels of $\mathrm{p}-\mathrm{AMPK} \alpha 2$ and AMPK $\alpha 2$ were normalized to GAPDH. WT, wild type; Ex, exercise. Values are mean \pm SD ( $n=6$ in each group). ${ }^{*} p<0.05 ;{ }^{* \star} p<0.01$ versus WT. ${ }^{* \star} p<0.001$ vs. WT+Ex. I indicates intima; M, media; A, adventitia.

four groups (Figure 2B), suggesting that exercise might have no effect on vasocontraction.

We then further investigated whether the difference in vasodilation was due to changes in eNOS/p-eNOS and the possible involvement of $\mathrm{AMPK} \alpha 2$. As expected, WT mice with exercise exhibited increased eNOS protein expression and phosphorylation in aorta compared with WT mice without exercise (Figure 2C). In contrast, $\mathrm{AMPK} \alpha 2^{-/-}$mice with exercise did not show any increase in total eNOS level or peNOS level in aorta compared with AMPK $\alpha 2^{-/-}$mice without exercise. These results indicated that the improved vasodilation of aortas during exercise training in mice might be through an AMPK $\alpha 2$-dependent mechanism.

\section{AMPK $\alpha 2$ Deficiency Results in Loss of Aortic Mitochondrial Content Increase with Exercise}

Accumulating studies indicated that mitochondrial content plays a critical role in maintaining vascular function. We thus evaluated whether exercise would have any effect on aortic mitochondrial content and the possible involvement of AMPK $\alpha 2$ by assessing mitochondrial fluorescence intensity, mtDNA copy number and Complex I protein expression.
As shown in Figure 3A, MitoTracker Green fluorescence intensity increased significantly in WT mice with exercise compared with WT mice without exercise. Consistently, the mtDNA copy number was upregulated by $34 \%$ in WT mice during exercise (Figure 3B). Meanwhile, Complex I protein expression also showed an increase of 2-folds in WT exercise mice compared to control mice (Figure 3C). In contrast, AMPK $\alpha 2^{-/-}$mice with exercise did not show any increase in mitochondrial fluorescence intensity, mtDNA copy number and Complex I protein compared with AMPK $\alpha 2^{-/-}$mice without exercise (Figure 3). These data suggested that exercise increased aortic mitochondrial content, and this effect was dependent on the presence of $\mathrm{AMPK} \alpha 2$.

\section{Effect of AMPK $\alpha 2$ Deficiency on Autophagy of Aortic Mitochondria with Exercise}

It has been reported that mitochondrial quantity and quality were controlled by biogenesis, fusion-fission, and autophagy. We therefore examined the effect of exercise on the expression of these relative proteins and the possible role of AMPK $\alpha 2^{-/-}$. We found that the expression of LC3B, an indicator of autophagy, and BNIP3L, a mitochondria-associated protein, were decreased in WT mice with exercise compared with WT mice without 


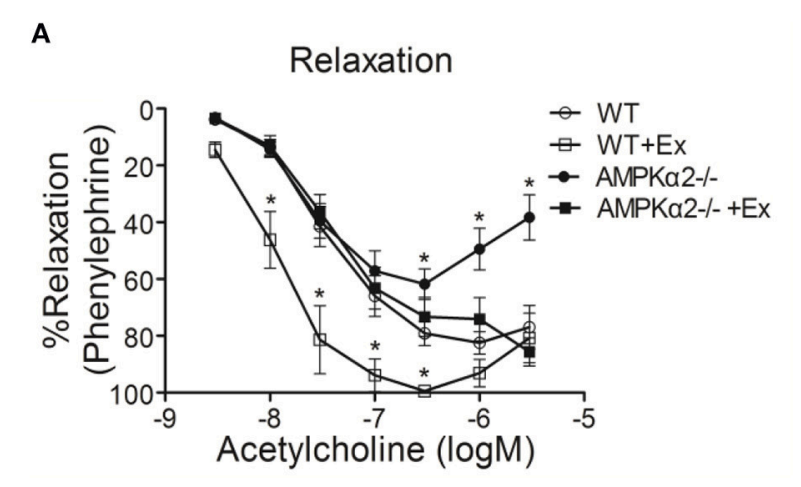

B
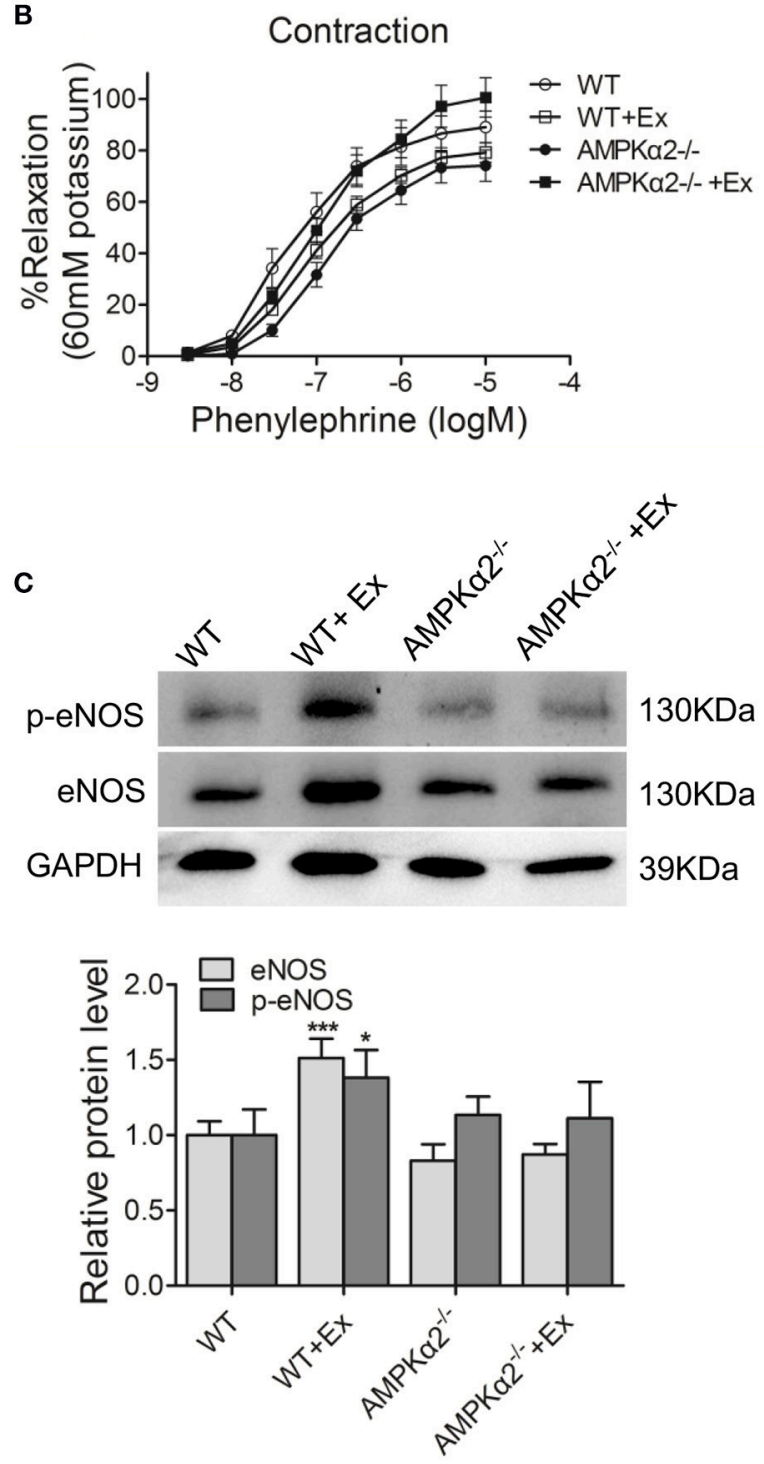

FIGURE 2 | Exercise improved vasodilation and endothelial function in an AMPK $\alpha 2$-dependent manner. (A) Dose-dependent vasorelaxation to acetylcholine of aorta from WT and AMPK $\alpha 2$ knockout mice with or without exercise. (B) Dose-dependent vasocontraction to phenylephrine of aorta from WT and AMPK $\alpha 2$ knockout mice with or without exercise. (C) Representative

(Continued)
FIGURE 2 | Continued

western blot images and summarized data showing the expression of p-eNOS and eNOS of aorta from WT and AMPK $\alpha 2$ knockout mice with or without exercise. The protein levels of $\mathrm{p}-\mathrm{eNOS}$ and eNOS were normalized to GAPDH. $W T$, wild type; Ex, exercise. Values are mean $\pm \mathrm{SD}\left(n=6\right.$ in each group). ${ }^{*} p<$ $0.05 ;{ }^{* \star *} p<0.001$ versus WT.

exercise (Figure 4B). Then we detected the protein expression of mTOR, the major autophagy negative regulator, and its phosphorylation at Ser2448. It was shown that WT exercise mice showed increased mTOR protein content compared to WT mice without exercise, but no significant alteration in phosphorylation activity (Figure 4B). In contrast, there was no significant difference in either LC3B/BNIP3L or mTOR protein levels in AMPK $\alpha 2^{-/-}$mice with exercise compared with these knockout mice without exercise. These results indicated that decreased autophagy may be responsible for exercise-related increase of mitochondrial content, and this effect was dependent on $\mathrm{AMPK} \alpha 2$.

In contrast, there was no significant difference of PGC- $1 \alpha$ (peroxisome proliferator-activated receptor gamma coactivator 1 alpha) protein expression, the main regulator of mitochondrial biogenesis, in the WT and AMPK $\alpha 2^{-/-}$mice with exercise compared with matched strain without exercise (Figure S2). Similarly, the protein levels of Drp1 (dynamin related protein 1) and Mfn1 (mitofusin 1), fission and fusion markers, also remained unchanged in the WT and AMPK $\alpha 2^{-/-}$mice with exercise compared with matched stains without exercise. (Figure S2). These results indicated that mitochondrial biogenesis, fission and fusion might not be involved in exercise-related mitochondria content increase in the aorta.

\section{AMPK $\alpha 2$ Deficiency Attenuates Exercise-Mediated Increase in Aortic Mitochondrial Antioxidant Capacity}

Finally, we evaluated the effect of exercise on mitochondrial antioxidant capacity and the possible involvement of AMPK $\alpha 2$. MnSOD and catalase are both critical to mitochondrial specific antioxidant defense. Figure 5A shows that the expression of catalase protein was significantly increased in the WT mice by $58 \%$ following exercise exposure, but not in $\mathrm{AMPK} \alpha 2^{-/-}$ mice. MnSOD protein content was significantly increased in WT mice after exercise intervention. Conversely, a marked decrease of MnSOD was observed in $\mathrm{AMPK} \alpha 2^{-/-}$mice with exercise compared with those without exercise (Figure 5A). These results indicated that exercise might increase mitochondrial antioxidant response in the aorta, and this effect was dependent on AMPK $\alpha 2$.

\section{DISCUSSION}

In the present study, we provide strong evidence that exercise promoted vasodilation, increased eNOS expression/ $/ 1177$. phosphorylation, and increased mitochondrial content and mitochondrial antioxidant capacity, resulting in increased vascular function. Importantly, these beneficial effects are dependent on the presence of AMPK $\alpha 2$ (Figure 5B). 
A
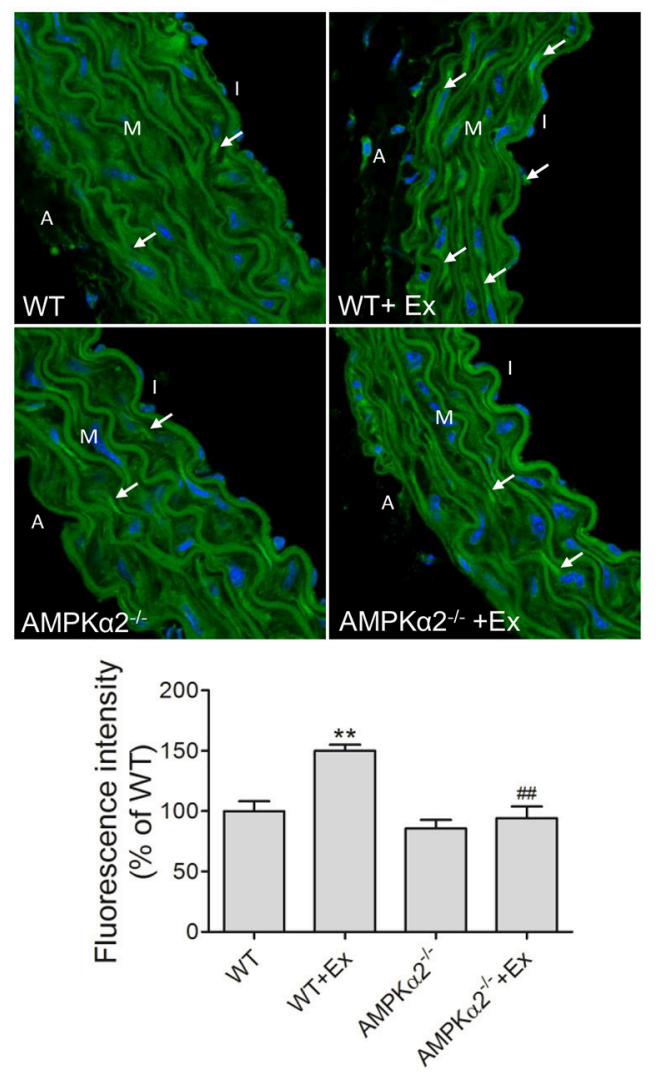

B
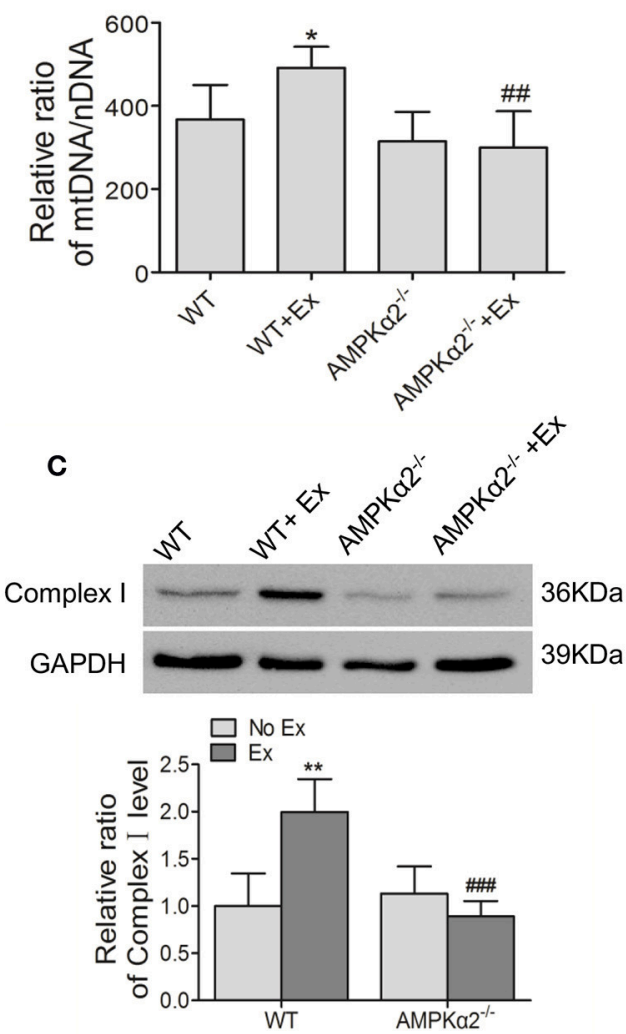

FIGURE 3 | Exercise training increased aortic mitochondrial content connected with AMPK $\alpha 2$. (A) Representative MitoTracker Green immunofluorescent images and fluorescence intensity analysis of aorta from WT and AMPKa2 knockout mice with or without exercise. Mitochondria were stained in green (arrow) and nuclei were counterstained with DAPI (blue). (B) Summarized data showing the mitochondria DNA copy number of aorta from WT and AMPK 22 knockout mice with or without exercise. (C) Representative western blot images and summarized data showing the expression of Complex I of aorta from WT and AMPK $\alpha 2$ knockout mice with or without exercise. The mitochondria DNA copy numbers were normalized to nuclear DNA, and the protein levels of Complex I were normalized to GAPDH. WT, wild type; Ex, exercise. Values are mean $\pm \mathrm{SD}\left(n=6\right.$ in each group). ${ }^{*} p<0.05 ;{ }^{\star *} p<0.01$ versus WT; \#\# $p<0.01 ; \# \# \# p<0.001$ versus WT+Ex. I indicates intima; $M$, media; $A$, adventitia.

Previous studies show that acute and long-term exercise training potently stimulate AMPK activity in skeletal muscles (Richter and Ruderman, 2009). Goirand et al. and Musi et al. have provided evidence that exercise training could activate cardiac and vascular AMPK in mice (Musi et al., 2005; Goirand et al., 2007). Consistent with these studies, the present study shows that exercise training significantly increased AMPK $\alpha 2$ activity in the vasculature. Furthermore, the present study shows that chronic exercise training increased vasodilation, eNOS expression and phosphorylation in aorta from mice, and these effects were lost in AMPK $\alpha 2$ knockout mice. It has been reported in a previous study that the activation of AMPK $\alpha 1$ was associated with increased vasodilatation and eNOS activation in mouse aorta during exercise training (Kröller-Schon et al., 2012). In this present study, we observed that $\mathrm{AMPK} \alpha 2$ activation in response to exercise-related protection also occurs in the vasculature. Moreover, acute exercise activated eNOS associated with the presence of AMPK in mouse aorta (Cacicedo et al., 2011), which is consistent with the present study showing that exercise increased eNOS activity through an AMPK $\alpha 2$ dependent manner.

Increasing evidence demonstrated that maintaining mitochondrial content/function and stability is essential to normal vascular systems (Dromparis and Michelakis, 2013; Kröller-Schon et al., 2013), and that exercise can stimulate key stress signals that control mitochondrial content and function in skeletal muscles (Russell et al., 2014). In the current study, we find that chronic exercise training induces an adaptive increase of mitochondrial quantity in the aorta, including the increased mtDNA and Complex I protein content. Further study shows that the expression of autophagy markers LC3B and BNIP3L (Zhu et al., 2013) was decreased, and that the expression of autophagy inhibitor marker mTOR (Kim et al., 2011) was increased in exercise training. These results indicate that autophagy was decreased in exercise and that the decreased autophagy might be responsible for the increased mitochondrial content in exercise. Consistent with the present study, it has been 

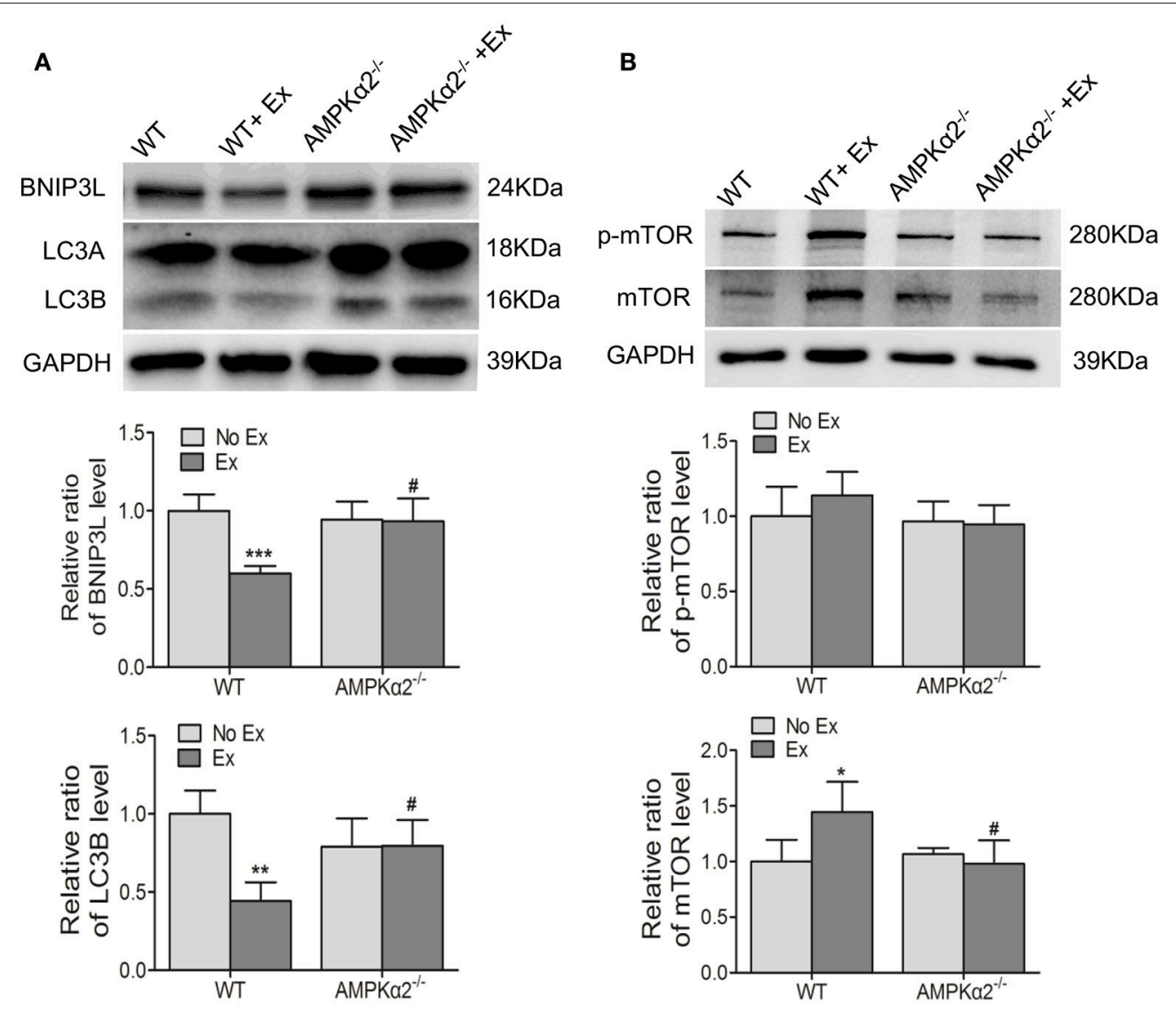

FIGURE 4 | The changes of mitochondria autophagy-related protein LC3B, BNIP3L, and mTOR during exercise training were dependent on AMPK $\alpha 2$. (A) Representative western blot images and summarized data showing the expression of LC3B and BNIP3L of aorta from WT and AMPK $\alpha 2$ knockout mice with or without exercise. (B) Representative western blot images and summarized data showing the expression of mTOR and p-mTOR of aorta from WT and AMPK 22 knockout mice with or without exercise. The protein levels of LC3B, BNIP3L, p-Mtor, and mTOR were normalized to GAPDH. WT, wild type; Ex, exercise. Values are mean $\pm \mathrm{SD}\left(n=6\right.$ in each group). ${ }^{*} p<0.05 ;{ }^{* *} p<0.01 ;{ }^{* \star *} p<0.001$ versus WT. \# $p<0.05$ versus WT+Ex.

shown that autophagy is decreased in skeletal muscle cells after exercise (Kim et al., 2012). Besides autophagy, mitochondria content can also be regulated by biogenesis, fission, and fusion (Youle and Narendra, 2011; Ding et al., 2013). In our study, mitochondrial biogenesis and mitochondrial fusion and fission may have no effect on the changes in aorta with exercise training, since the expression of PGC-1 $\alpha, \mathrm{Mfn} 1$, and Drp1 was not changed in exercised mice compared mice without exercise. In contrast, previous studies suggest that AMPK works through PGC- $1 \alpha$ to promote mitochondrial biogenesis in acute exercise in skeletal muscles (Kahn et al., 2005; Reznick and Shulman, 2006), and that endurance training increases Mfn1 content to induce mitochondrial fusion in rat liver (Gonçalves et al., 2016). These results suggest that mitochondrial content may be regulated through different signaling pathways in different cells in exercise training.

Furthermore, our study demonstrates that chronic exercise training upregulated the protein expression of MnSOD and catalase, and the increases are depended on the AMPK $\alpha 2$ isoform. Indeed, it has been reported that antioxidant enzymes were activated by exercise training in rat brains (Marosi et al., 2012; Marcelino et al., 2013), and chronic aerobic exercise training increased aortic mitochondrial antioxidant enzyme in aged rats ( $\mathrm{Gu}$ et al., 2014). Consistent with our results, $\mathrm{AMPK} \alpha 2$ has been reported to be involved in the protective effect of swimming training against isoproterenol-induced ROS production and promote the expression of antioxidant enzymes in mouse hearts (Ma et al., 2015). AMPK activity has been reported to be associated with the redox reaction in different tissues in the cardiovascular system (Ma et al., 2015). Activation of AMPK by 5-aminoimidazole-4-carboxamide ribonucleotide (AICAR) could significantly decrease ROS which was induced by palmitic acid in human aortic endothelial cells and increased expression of the antioxidant thioredoxin (Li et al., 2009). In addition, $\mathrm{AMPK} \alpha 2$ suppressed NADPH oxidase expression and reduced ROS production in endothelial cells (Wang et al., 2010) as well as induced manganese SOD (Kukidome et al., 2006). 

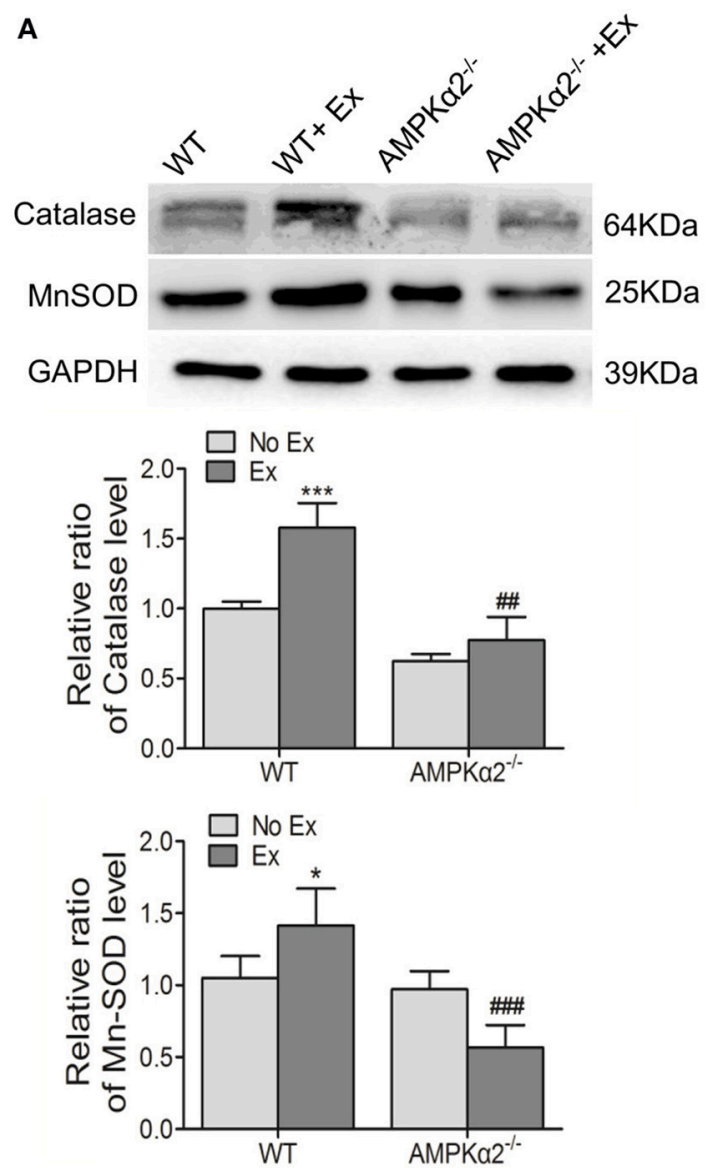

B

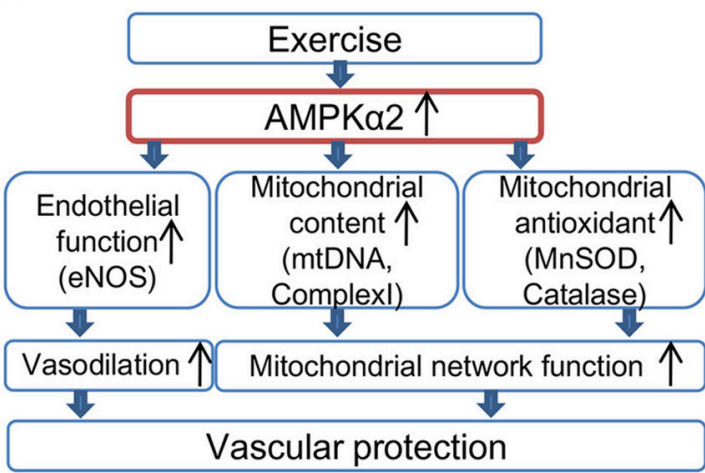

FIGURE 5 | Exercise training enhanced aortic mitochondrial MnSOD and catalase via AMPK $\alpha 2$. (A) Representative western blot images and summarized data showing the expression of MnSOD and catalase of aorta from WT and AMPK $\alpha 2$ knockout mice with or without exercise. (B) Scheme illustrates that AMPK $\alpha 2$ plays a critical role in exercise-related vascular protection via increasing endothelial and mitochondrial function in the artery. AMPKa2 is activated after chronic exercise training and in turn, mediates increased eNOS expression and activation, increased mitochondrial content (mtDNA and Complex I) and mitochondrial antioxidant capacity (MnSOD and catalase). These events contribute to the promoted vasodilation and mitochondrial network function, resulting in improved vascular function. The protein levels of MnSOD and catalase were normalized to GAPDH. WT, wild type; Ex, exercise. Values are mean $\pm \mathrm{SD}\left(n=6\right.$ in each group). ${ }^{*} p<0.05$; $p<0.001$ versus WT. \#\# $p<0.01 ; \# \# \# p<0.001$ versus $W T+E x$.
Finally, our results show that exercise-related increases of eNOS expression/phosphorylation, mitochondrial content and $\mathrm{MnSOD} /$ catalase expression were lost in AMPK $\alpha 2$ knockout mice. As demonstrated in the present study, the increased vasodilation and eNOS activation during exercise were decreased in AMPK $\alpha 2$ deficient mice. This may be caused by the finding that AMPK is able to phosphorylate eNOS, thereby leading to eNOS activation and increased NO production (Morrow et al., 2003), and that the loss of AMPKa2 may prevent exercise-related eNOS activation. As for the increased expression of eNOS, mitochondria content and increased expression of MnSOD and catalase in aorta with exercise, the increased AMPK $\alpha 2$ may induce the expression of these proteins through direct interaction with the nucleus in WT mice as previously reported (McGee et al., 2003; Jørgensen et al., 2006). In AMPK $\alpha 2$ knockout mice, however, these corresponding proteins were not increased due to the lack of AMPK $\alpha 2$ to promote the expression of these proteins. Indeed, a recent report showed that AMPK signaling is required for the metabolic response to exercise in vivo, and AMPK activation was proposed as a regulatory mechanism that underlies exercise-induced glucose uptake in muscles, thereby leading to increased systemic insulin sensitivity (Steinberg and Jørgensen, 2007).

It has been shown that AMPK plays a protective role in diabetes and hypertension. For example, Wang et al. reported that AMPK activation was reduced in diabetic mice, and that metformin, an AMPK activator, normalized the acetylcholine-induced endothelial relaxation (Wang et al., 2009). Sun et al. found that resveratrol, a compound that activates AMPK, induced vasodilation and lowered blood pressure in DOCA-hypertensive mice(Sun et al., 2015). Metformin attenuated cytokine-induced expression of proinflammatory factors via AMPK activation in human umbilical vein endothelial cells (Hattori et al., 2006). The present finding that exercise improved aortic endothelial and mitochondrial function via AMPK $\alpha 2$ activation suggests that AMPK $\alpha 2$ may play a critical role in exerciserelated improvement of vascular function in diabetes and hypertension.

In summary, our study shows that chronic exercise training mediates vascular protection through improving aortic endothelial and mitochondrial function, and that vascular AMPK isoform AMPK $\alpha 2$ is a key signaling molecule that mediates the protective effects of exercise in the vasculature. These findings may provide a novel mechanism in exercise-related cardiovascular protection.

\section{AUTHOR CONTRIBUTIONS}

XC designed the study, performed the experiments, collected and analyzed the data, and wrote and revised the final version of the manuscript. XA and DC contributed to data collection. WS, YZ, and PG designed the study. MY, WH, and PG critically revised the final version of the manuscript. All authors read and approved the final version of the manuscript. 


\section{FUNDING}

This study was supported by the National Natural Science Foundation of China $(81100184,81230071,81300089,81200203$, 91539202, and 81570221), the Scientific Fund of Shanghai Jiao Tong University School of Medicine (14XJ10042), the Pujiang Program of the Shanghai Science and Technology Committee (14PJ1406400), the Scientific Research Foundation for the Returned Overseas Chinese Scholars of the State Education Ministry, and the Shanghai Medical Bureau Fund (201540037).

\section{REFERENCES}

Adams, V., Linke, A., Kränkel, N., Erbs, S., Gielen, S., Möbius-Winkler, S., et al. (2005). Impact of regular physical activity on the $\mathrm{NAD}(\mathrm{P}) \mathrm{H}$ oxidase and angiotensin receptor system in patients with coronary artery disease. Circulation 111, 555-562. doi: 10.1161/01.CIR.0000154560.88933.7E

Cacicedo, J. M., Gauthier, M. S., Lebrasseur, N. K., Jasuja, R., Ruderman, N. B., and Ido, Y. (2011). Acute exercise activates AMPK and eNOS in the mouse aorta. Am. J. Physiol. Heart Circ. Physiol. 301, H1255-H1265. doi: 10.1152/ajpheart.01279.2010

Dikalova, A. E., Bikineyeva, A. T., Budzyn, K., Nazarewicz, R. R., McCann, L., Lewis, W., et al. (2010). Therapeutic targeting of mitochondrial superoxide in hypertension. Circ. Res. 107, U106-U221. doi: 10.1161/CIRCRESAHA.109.214601

Ding, H., Zhang, Z. Y., Zhang, J. W., and Zhang, Y. (2013). Role of mitochondrial quality control in exercise-induced health adaptation. Zhongguo Ying Yong Sheng Li Xue Za Zhi 29, 543-553.

Dromparis, P., and Michelakis, E. D. (2013). Mitochondria in vascular health and disease. Annu. Rev. Physiol. 75, 95-126. doi: 10.1146/annurev-physiol-030212-183804

Fernando, P., Bonen, A., and Hoffman-Goetz, L. (1993). Predicting submaximal oxygen consumption during treadmill running in mice. Can. J. Physiol. Pharmacol. 71, 854-857. doi: 10.1139/y93-128

Goirand, F., Solar, M., Athea, Y., Viollet, B., Mateo, P., Fortin, D., et al. (2007). Activation of AMP kinase $\alpha 1$ subunit induces aortic vasorelaxation in mice. J. Physiol. 581(Pt 3), 1163-1171. doi: 10.1113/jphysiol.2007.132589

Gonçalves, I. O., Passos, E., Diogo, C. V., Rocha-Rodrigues, S., SantosAlves, E., Oliveira, P. J., et al. (2016). Exercise mitigates mitochondrial permeability transition pore and quality control mechanisms alterations in nonalcoholic steatohepatitis. Appl. Physiol. Nutr. Metab. 41, 298-306. doi: 10.1139/apnm-2015-0470

Gu, Q., Wang, B., Zhang, X. F., Ma, Y. P., Liu, J. D., and Wang, X. Z. (2014). Chronic aerobic exercise training attenuates aortic stiffening and endothelial dysfunction through preserving aortic mitochondrial function in aged rats. Exp. Gerontol. 56, 37-44. doi: 10.1016/j.exger.2014.02.014

Hambrecht, R., Adams, V., Erbs, S., Linke, A., Kränkel, N., Shu, Y., et al. (2003). Regular physical activity improves endothelial function in patients with coronary artery disease by increasing phosphorylation of endothelial nitric oxide synthase. Circulation 107, 3152-3158. doi: 10.1161/01.CIR.0000074229.93804.5C

Hambrecht, R., Wolf, A., Gielen, S., Linke, A., Hofer, J., Erbs, S., et al. (2000). Effect of exercise on coronary endothelial function in patients with coronary artery disease. N. Engl. J. Med. 342, 454-460. doi: 10.1056/NEJM200002173420702

Hattori, Y., Suzuki, K., Hattori, S., and Kasai, K. (2006). Metformin inhibits cytokine-induced nuclear factor $\kappa \mathrm{B}$ activation via AMP-activated protein kinase activation in vascular endothelial cells. Hypertension 47, 1183-1188. doi: 10.1161/01.HYP.0000221429.94591.72

Jin, H. S., Sober, S., Hong, K. W., Org, E., Kim, B. Y., Laan, M., et al. (2011). Age-dependent association of the polymorphisms in the mitochondria-shaping gene, OPA1, with blood pressure and hypertension in Korean population. Am. J. Hypertens. 24, 1127-1135. doi: 10.1038/ajh.2011.131
ACKNOWLEDGMENTS

Thank you to all the participants who were involved in the research. There are no conflicts of interest to disclose.

\section{SUPPLEMENTARY MATERIAL}

The Supplementary Material for this article can be found online at: http://journal.frontiersin.org/article/10.3389/fphys. 2016.00631/full\#supplementary-material

Jørgensen, S. B., Richter, E. A., and Wojtaszewski, J. F. (2006). Role of AMPK in skeletal muscle metabolic regulation and adaptation in relation to exercise. $J$. Physiol. Lond. 574, 17-31. doi: 10.1113/jphysiol.2006.109942

Kahn, B. B., Alquier, T., Carling, D., and Hardie, D. G. (2005). AMP-activated protein kinase: ancient energy gauge provides clues to modern understanding of metabolism. Cell Metab. 1, 15-25. doi: 10.1016/j.cmet.2004.12.003

Kim, J., Kundu, M., Viollet, B., and Guan, K. L. (2011). AMPK and mTOR regulate autophagy through direct phosphorylation of Ulk1. Nat. Cell Biol. 13, U132-U171. doi: 10.1038/ncb2152

Kim, Y. A., Kim, Y. S., and Song, W. (2012). Autophagic response to a single bout of moderate exercise in murine skeletal muscle. J. Physiol. Biochem. 68, 229-235. doi: 10.1007/s13105-011-0135-x

Kojda, G., Cheng, Y. C., Burchfield, J., and Harrison, D. G. (2001). Dysfunctional regulation of endothelial nitric oxide synthase (eNOS) expression in response to exercise in mice lacking one eNOS gene. Circulation 103, 2839-2844. doi: 10.1161/01.CIR.103.23.2839

Kröller-Schon, S., Jansen, T., Hauptmann, F., Schüler, A., Heeren, T., Hausding, M., et al. (2012). a1AMP-activated protein kinase mediates vascular protective effects of exercise. Arterioscler. Thromb. Vasc. Biol. 32, 1632-1641. doi: 10.1161/ATVBAHA.111.243980

Kröller-Schon, S., Jansen, T., Schüler, A., Oelze, M., Wenzel, P., Hausding, M., et al. (2013). Peroxisome proliferator-activated receptor gamma, coactivator $1 \alpha$ deletion induces angiotensin II-associated vascular dysfunction by increasing mitochondrial oxidative stress and vascular inflammation. Arterioscler. Thromb. Vasc. Biol. 33, 1928-1935. doi: 10.1161/ATVBAHA.113.301717

Kukidome, D., Nishikawa, T., Sonoda, K., Imoto, K., Fujisawa, K., Yano, M., et al. (2006). Activation of AMP-activated protein kinase reduces hyperglycemiaInduced mitochondrial reactive oxygen species production and promotes mitochondrial biogenesis in human umbilical vein endothelial cells. Diabetes 55, 120-127. doi: 10.2337/diabetes.55.01.06.db05-0943

Kumral, Z. N., Sener, G., Ozgur, S., Koc, M., Suleymanoglu, S., Hurdag, C., et al. (2016). Regular exercise alleviates renovascular hypertension-induced cardiac/endothelial dysfunction and oxidative injury in rats. J. Physiol. Pharmacol. 67, 45-55.

Li, X. D., Chen, J., Ruan, C. C., Zhu, D. L., and Gao, P. J. (2012). Vascular endothelial growth factor-induced osteopontin expression mediates vascular inflammation and neointima formation via Flt-1 in adventitial fibroblasts. Arterioscler. Thromb. Vasc. Biol. 32, 2250-2258. doi: 10.1161/ATVBAHA.112.255216

Li, X. N., Song, J., Zhang, L., LeMaire, S. A., Hou, X. Y., Zhang, C., et al. (2009). Activation of the AMPK-FOXO3 pathway reduces fatty acid-induced increase in intracellular reactive oxygen species by upregulating thioredoxin. Diabetes 58, 2246-2257. doi: 10.2337/db08-1512

Ma, X. W., Fu, Y. N., Xiao, H., Song, Y., Chen, R. F., Shen, J., et al. (2015). Cardiac fibrosis alleviated by exercise training is AMPK-dependent. PLoS ONE 10:e0129971. doi: 10.1371/journal.pone.0129971

Magnoni, L. J., Palstra, A. P., and Planas, J. V. (2014). Fueling the engine: induction of AMP-activated protein kinase in trout skeletal muscle by swimming. J. Exp. Biol. 217(Pt 10), 1649-1652. doi: 10.1242/jeb.099192

Marcelino, T. B., Longoni, A., Kudo, K. Y., Stone, V., Rech, A., de Assis, A. M., et al. (2013). Evidences that maternal swimming exercise improves antioxidant 
defenses and induces mitochondrial biogenesis in the brain of young wistar rats. Neuroscience 246, 28-39. doi: 10.1016/j.neuroscience.2013.04.043

Marosi, K., Bori, Z., Hart, N., Sárga, L., Koltai, E., Radák, Z., et al. (2012). Longterm exercise treatment reduces oxidative stress in the hippocampus of aging rats. Neuroscience 226, 21-28. doi: 10.1016/j.neuroscience.2012.09.001

McGee, S. L., Howlett, K. F., Starkie, R. L., Cameron-Smith, D., Kemp, B. E., and Hargreaves, M. (2003). Exercise increases nuclear AMPK $\alpha(2)$ in human skeletal muscle. Diabetes 52, 926-928. doi: 10.2337/diabetes.52.4.926

Morrow, V. A., Foufelle, F., Connell, J. M., Petrie, J. R., Gould, G. W., and Salt, I. P. (2003). Direct activation of AMP-activated protein kinase stimulates nitric-oxide synthesis in human aortic endothelial cells. J. Biol. Chem. 278, 31629-31639. doi: 10.1074/jbc.M212831200

Musi, N., Hirshman, M. F., Arad, M., Xing, Y., Fujii, N., Pomerleau, J., et al. (2005). Functional role of AMP-activated protein kinase in the heart during exercise. FEBS Lett. 579, 2045-2050. doi: 10.1016/j.febslet.2005.02.052

Nagata, D., Takeda, R., Sata, M., Satonaka, H., Suzuki, E., Nagano, T., et al. (2004). AMP-activated protein kinase inhibits angiotensin II-stimulated vascular smooth muscle cell proliferation. Circulation 110, 444-451. doi: 10.1161/01.CIR.0000136025.96811.76

Pangare, M., and Makino, A. (2012). Mitochondrial function in vascular endothelial cell in diabetes. J. Smooth Muscle Res. 48, 1-26. doi: 10.1540/jsmr.48.1

Ray Hamidie, R. D., Yamada, T., Ishizawa, R., Saito, Y., and Masuda, K. (2015). Curcumin treatment enhances the effect of exercise on mitochondrial biogenesis in skeletal muscle by increasing cAMP levels. Metab. Clin. Exp. 64, 1334-1347. doi: 10.1016/j.metabol.2015.07.010

Reznick, R. M., and Shulman, G. I. (2006). The role of AMP-activated protein kinase in mitochondrial biogenesis. J. Physiol. 574( $\mathrm{Pt} \mathrm{1),} \mathrm{33-39.}$ doi: 10.1113/jphysiol.2006.109512

Richter, E. A., and Ruderman, N. B. (2009). AMPK and the biochemistry of exercise: implications for human health and disease. Biochem. J. 418, 261-275. doi: 10.1042/BJ20082055

Russell, A. P., Foletta, V. C., Snow, R. J., and Wadley, G. D. (2014). Skeletal muscle mitochondria: a major player in exercise, health and disease. Biochim. Biophys. Acta 1840, 1276-1284. doi: 10.1016/j.bbagen.2013.11.016

Salabei, J. K., and Hill, B. G. (2013). Mitochondrial fission induced by plateletderived growth factor regulates vascular smooth muscle cell bioenergetics and cell proliferation. Redox Biol. 1, 542-551. doi: 10.1016/j.redox.2013.10.011

Schulz, E., Dopheide, J., Schuhmacher, S., Thomas, S. R., Chen, K., Daiber, A., et al. (2008). Suppression of the JNK pathway by induction of a metabolic stress response prevents vascular injury and dysfunction. Circulation 118, 1347-1357. doi: 10.1161/CIRCULATIONAHA.108.784289

Steinberg, G. R., and Jørgensen, S. B. (2007). The AMP-activated protein kinase: role in regulation of skeletal muscle metabolism and insulin sensitivity. Mini Rev. Med. Chem. 7, 519-526. doi: 10.2174/138955707780619662

Steinberg, G. R., and Kemp, B. E. (2009). AMPK in health and disease. Physiol. Rev. 89, 1025-1078. doi: 10.1152/physrev.00011.2008
Stewart, K. J. (2002). Exercise training and the cardiovascular consequences of type 2 diabetes and hypertension: plausible mechanisms for improving cardiovascular health. JAMA 288, 1622-1631. doi: 10.1001/jama.288.13.1622

Sun, G. Q., Li, Y. B., Du, B., and Meng, Y. (2015). Resveratrol via activation of AMPK lowers blood pressure in DOCA-salt hypertensive mice. Clin. Exp. Hypertens 37, 616-621. doi: 10.3109/10641963.2015.1036060

Szewczyk, A., Jarmuszkiewicz, W., Koziel, A., Sobieraj, I., Nobik, W., Lukasiak, A., et al. (2015). Mitochondrial mechanisms of endothelial dysfunction. Pharmacol. Rep. 67, 704-710. doi: 10.1016/j.pharep.2015.04.009

Tang, X., Luo, Y. X., Chen, H. Z., and Liu, D. P. (2014). Mitochondria, endothelial cell function, and vascular diseases. Front. Physiol. 5:175. doi: 10.3389/fphys.2014.00175

Wang, S., Xu, J., Song, P., Viollet, B., and Zou, M. H. (2009). In vivo activation of AMP-activated protein kinase attenuates diabetes-enhanced degradation of GTP cyclohydrolase I. Diabetes 58, 1893-1901. doi: 10.2337/db09-0267

Wang, S., Zhang, M., Liang, B., Xu, J., Xie, Z., Liu, C., et al. (2010). AMPK $\alpha 2$ deletion causes aberrant expression and activation of $\mathrm{NAD}(\mathrm{P}) \mathrm{H}$ oxidase and consequent endothelial dysfunction in vivo: role of $26 \mathrm{~S}$ proteasomes. Circ. Res. 106, 1117-1128. doi: 10.1161/CIRCRESAHA.109.212530

Wenzel, P., Schuhmacher, S., Kienhöfer, J., Müller, J., Hortmann, M., Oelze, M., et al. (2008). Manganese superoxide dismutase and aldehyde dehydrogenase deficiency increase mitochondrial oxidative stress and aggravate age-dependent vascular dysfunction. Cardiovasc. Res. 80, 280-289. doi: 10.1093/cvr/cvn182

Yan, Z., Lira, V. A., and Greene, N. P. (2012). Exercise training-induced regulation of mitochondrial quality. Exerc. Sport Sci. Rev. 40, 159-164. doi: $10.1097 /$ jes.0b013e3182575599

Youle, R. J., and Narendra, D. P. (2011). Mechanisms of mitophagy. Nat. Rev. Mol. Cell Biol. 12, 9-14. doi: 10.1038/nrm3028

Zhou, W., Hong, M., Zhang, K., Chen, D., Han, W., Shen, W., et al. (2014). Mechanisms of improved aortic stiffness by arotinolol in spontaneously hypertensive rats. PLoS ONE 9:e88722. doi: 10.1371/journal.pone.0088722

Zhu, Y. Y., Massen, S., Terenzio, M., Lang, V., Chen-Lindner, S., Eils, R., et al. (2013). Modulation of serines 17 and 24 in the LC3-interacting region of Bnip3 determines pro-survival mitophagy versus apoptosis. J. Biol. Chem. 288, 1099-1113. doi: 10.1074/jbc.M112.399345

Conflict of Interest Statement: The authors declare that the research was conducted in the absence of any commercial or financial relationships that could be construed as a potential conflict of interest.

Copyright (c) 2016 Chen, An, Chen, Ye, Shen, Han, Zhang and Gao. This is an open-access article distributed under the terms of the Creative Commons Attribution License (CC BY). The use, distribution or reproduction in other forums is permitted, provided the original author(s) or licensor are credited and that the original publication in this journal is cited, in accordance with accepted academic practice. No use, distribution or reproduction is permitted which does not comply with these terms. 